\title{
Device-centric Spectrum Management
}

\author{
Haitao Zheng \\ Department of Computer Science \\ University of California, Santa Barbara, U.S.A \\ Email: htzheng@cs.ucsb.edu
}

\author{
Lili Cao \\ Department of Computer Science \\ Shanghai Jiaotong University, Shanghai, P.R. China \\ Email: cao_li_li@yahoo.com
}

\begin{abstract}
Efficient spectrum allocation in Open Spectrum systems is a challenging problem, particularly for devices with constrained communication resources such as sensor and mobile ad hoc networks. We propose a device-centric spectrum management scheme with low communication costs, where users observe local interference patterns and act independently according to preset spectrum rules. We propose five rules that tradeoff performance with implementation complexity and communication costs, and derive a lower bound on each user's allocation based on these rules. Experimental results show that our proposed rule-based approach reduces communication costs from efficient collaborative approaches by a factor of 3-4 while providing good performance.
\end{abstract}

\section{INTRODUCTION}

Wireless radio spectrum is a precious finite resource. To eliminate interference, traditional spectrum management schemes license fixed spectrum slices to each wireless technology. While new devices demand spectrum access, most of the existing spectrum is claimed, and often under-utilized, by legacy devices. Fixed assignment is clearly non-optimal. While studies find more than $70 \%$ of spectrum is unutilized in most areas [15], reusing this spectrum will solve today's spectrum scarcity problem.

One solution is the use of "open spectrum systems" [2], [17] enabled by advances in cognitive radio [4], [14]. While legacy license holders or primary users have priority in spectrum access, unlicensed users or secondary users opportunistically use available spectrum without interfering with primary users. The result provides efficient spectrum usage and simplifies deployment of new applications.

A critical problem in open spectrum systems is efficient spectrum management for secondary users. While maximizing utilization is the primary goal, a good management scheme also needs to minimize interference and provide a degree of fairness across users. Our previous work on decentralized spectrum allocation [6], [18], [25] has shown that user collaboration leads to results that closely approximate the optimal allocation. Collaboration in this context requires users to be somewhat selfless, occasionally sacrificing local performance to improve overall system utility. It also requires coordination and frequent information exchange among users.

We observe that a collaborative model may heavily stress the communication resources of constrained networks such as sensor and mobile ad hoc networks. In this paper, we propose an alternative device-centric spectrum management scheme where users act independently based on local observations of interference patterns and neighboring devices. This approach greatly simplifies allocation and significantly reduces control traffic.

This paper makes three contributions. First, we propose a management system where users act based on local observations. We specify five rules that regulate users' spectrum access to tradeoff fairness and utilization with communication costs and algorithm complexity. Second, we prove that a system under these rules converges to equilibrium in finite iterations, and derive a lower bound on each user's spectrum allocation. Finally, we use extensive simulations to quantify the impact of these spectrum rules on network access, while comparing the rule based and collaboration based approaches in efficiency and complexity.

The rest of the paper is organized as follows. We begin in Section II by describing the spectrum allocation problem and summarizing the existing solutions. Next, we propose the rule-based spectrum management system in Section III and develop specific spectrum rules in Section IV. In Section V, we conduct experiments to compare the rules-based approaches to the collaboration-based approaches. We discuss future work in Section VI and conclude in Section VII.

\section{BACKGROUND AND RELATED WORK}

In this section, we briefly introduce the background of open spectrum systems and existing work.

\section{A. Background}

Open spectrum focuses on controlling the behavior of secondary users while keeping the system transparent to primary users. Channels are the fundamental units of spectrum usage. The operational spectrum is partitioned into non-overlapping channels by one or a combination of time-, frequency-, or code-division. Each secondary user conducts spectrum sensing to identify and track its spectrum opportunities, i.e. the set of channels it can transmit successfully on without interfering with existing primary users. Since spectrum opportunity depends on neighboring primary users' spectrum usage, secondary users observe spectrum heterogeneity, i.e. spectrum availability fluctuates over time and location.

Spectrum management coordinates secondary users' channel usage to prevent conflicts between secondary users and both primary and other secondary users, while promoting utilization and fairness. Spectrum management can be reduced into a constrained channel allocation problem that accounts 
for heterogeneous spectrum availability and possible conflicts at each user. In addition to maximizing spectrum utilization and fairness, a solution must provide a feasible channel usage enforcement, e.g. a conflict free channel assignment in order to deploy the network.

Spectrum management in open spectrum systems is different from management in voice and general wireless networks. Where the objective in voice networks is to support as many homogeneous flows, open spectrum users have varying bandwidth requirements, and fairness is essential to provide each user with a feasible packet delivery rate. While the general channel allocation problem has been well studied for wireless networks, particularly for cellular and multi-hop ad hoc networks, it is possible to apply some existing solutions to the new problem. However, as we will describe in Section II-B, existing work on channel allocation has different objective and applicable scenario from those in open spectrum systems. New solutions are necessary to successfully deploy open spectrum systems.

Note that in this paper, we only consider conflicts due to interference from neighboring transmissions. Time-based conflicts from multiple actions in the same time slot, such as receiving data simultaneously from two transmitters or transmitting to multiple receivers, has been addressed in prior work [21], [22]. Resolving this requires coordination between the sender and receiver, and a distributed solution is provided in [24].

\section{B. Existing Work on Channel Allocation}

Optimal conflict-free channel assignment satisfying a global optimal objective is often NP-hard, even when global topology information is available [7]. Centralized approximations are widely used in single hop wireless networks such as cellular networks. This can be easily extended to multi-hop wireless networks by flooding connectivity and traffic requirements across the network, and requiring all users to run a variant of the centralized algorithm. However, this approach clearly does not scale as networks become larger and more dynamic.

An alternative decentralized allocation, where users act based on locally available information is much more attractive. Both analytical framework and practical strategies have been proposed. Analytical frameworks in [10], [16] address fairness for single-hop flows, and derive an estimate of the rate at each flow to achieve Max-Min fairness. However, there is no guarantee that a feasible scheme exists to achieve the rate.

Practical strategies have been proposed for sharing a single channel. Contention based schemes invoke a random access protocol like ALOHA and CSMA, where users contend in time to share a common channel [10], [13], [16]. While this scheme provides fairness and utilization on a single channel system probabilistically, its application to a multi-channel system requires each user to know how many and which channel(s) to access. Another approach, conflict free time slot scheduling, provides guaranteed channel usage by reserving time slots for each flow. Solutions in [1], [21], [20] assign exactly one time slot to each flow. This approach can be used in multichannel systems if each user uses only one channel. Another solution [22] allows users to use multiple slots/channels to achieve Max-Min-fair, but does not consider interference from neighbor transmissions.

Multi-channel assignment strategies were developed mostly for cellular networks. The work in [12] provides solutions to assign frequency bands among base stations to minimize call blocking probability for voice traffic. There is no notion of fairness as the traffic determines the number of channels each base station should use. Distributed channel assignment for OFDM based systems has been studied in [8] but only for single hop network, where all the flows interfere with each other.

From the above, we see that although efficient strategies exist to allocate channel(s) to users, they do not address the nature of spectrum heterogeneity in multi-channel, multihop network topology, and the problem of assigning multiple channels to each secondary user to maximize fairness. Hence, the associated channel allocation problem in open spectrum systems requires new solutions.

\section{Existing Work on Spectrum Management}

Previous work in this context took a collaborative approach, where secondary users negotiated spectrum with neighbors in order to maximize system utility, as defined by optimization objectives such as fairness and utilization [6], [18], [25]. [18] and [25] reduce the problem to a variant of graph coloring problem and propose a labelling scheme to prioritize users in channel assignment. This allocation is optimized for a fixed topology, and needs to be refreshed up on topology changes. In [6], we propose a local bargaining approach where users negotiate spectrum assignment within local self-organized groups. The algorithm provides a fair service guarantee (defined by Poverty Line) for each user while significantly reducing the communication overhead in allocation.

These collaboration-based approaches share the property that neighbors exchange coordination information frequently. This requires both a common coordination protocol and a communication path, resulting in implementation complexity and communication overhead. While practical for users not constrained in energy or bandwidth, such as access points and broadcast stations, they are not ideal for resource constrained secondary users. That problem is addressed in this paper.

\section{Rule Based Spectrum ManAGEMENT}

For energy-constrained devices such as sensors and mobile ad hoc devices, frequent communication between devices is undesirable. In addition, future networks are likely to have heterogeneous devices and access technologies [3], making the implementation of a common coordination protocol a significant challenge. For these types of networks, we propose a device-centric management scheme, where users observe local conditions and neighbors' actions, and independently adapt their spectrum usage using a set of rules predefined by spectrum regulators. In contrast to a collaborative approach [6], 


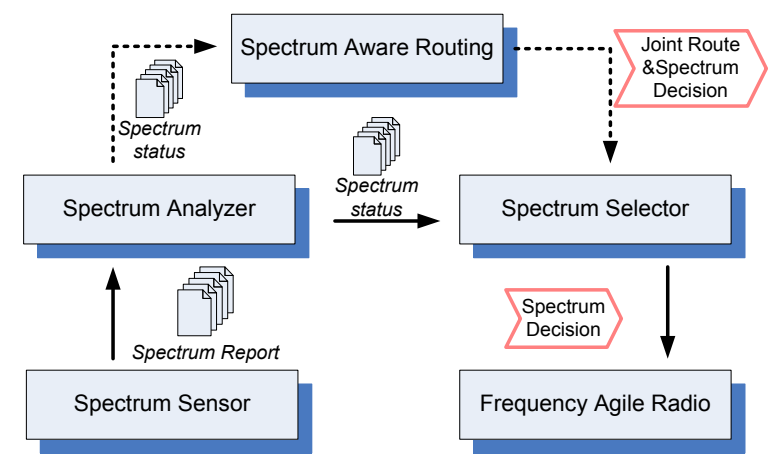

Fig. 1. Spectrum Management System on Each Secondary Device

[18], [25], users tend to prioritize their own performance with minimal regard to system utility. Their compliance with the rules promotes efficient and fair spectrum sharing.

In our work, each secondary device has the following components. First, a spectrum sensor monitors spectrum usage in the neighborhood and produces periodic reports. Next, a spectrum analyzer examines the report to identify primary users and available spectrum based on local configurations. Then the user's spectrum selector follows the spectrum rules to choose a set of appropriate spectrum bands. Any environment change such as user movement or traffic variation might trigger an adjustment. Finally, the frequency agile radio module reconfigures RF to switch to the newly selected frequencies, and uses the appropriate protocol and modulation on each frequency. Note that we do not require the selected frequencies/channels to be contiguous, and it is assumed that the radio can send packets over non-contiguous frequencies [23]. It should be noted that modifications are required at higher layers to respond to dynamically changing spectrum availability among secondary users. For example, a spectrum aware routing protocol adapts route selection to spectrum fluctuations. Figure 1 illustrates the architecture sketch of the proposed framework.

In this paper, we focus on the spectrum selector component and the rules that guide channel selection. Approaches to the other components either exist or are under current study. Consistent with prior work [10], [13], [16], [22], we consider and address fairness for single-hop flows, since they are the simplest format in wireless transmissions. Finally, we postpone the discussion of spectrum-aware routing protocols to a future paper.

Figure 2 describes the operation of the spectrum selector component. The spectrum status is read in, and checked against the spectrum rules. If the user is satisfied, no change is necessary and the selector terminates. Otherwise, the rules are used to determine the appropriate channels, and the spectrum decision are made accordingly. The spectrum assignment needs to maximize utilization, minimize interference, and provide a degree of fairness across users. For fairness, we use the proportional fairness metric [16] commonly used in communication systems. The spectrum rules are the key to making these tradeoffs, and will be discussed in detail in

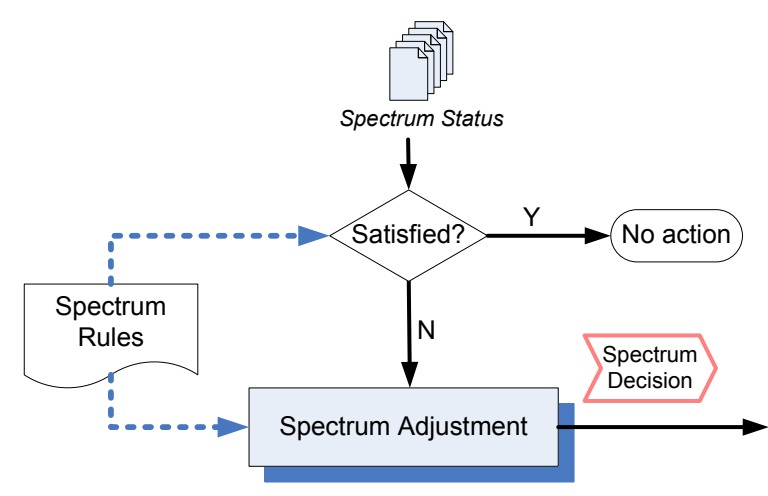

Fig. 2. Spectrum Selector Structure

Section IV.

\section{Spectrum Management Rules}

The spectrum rules specify tradeoffs between performance and signaling complexity according to each application scenario. In this section, we propose five different rules and provide theoretical insights on each rule's performance.

\section{A. Network Model}

We first describe our notations and assumptions. We consider a network with some primary users and $N$ secondary users. The spectrum is partitioned to $M$ orthogonal noninterfering channels. Given primary users' spectrum usage, each secondary user $n$ keeps track a list of channels available for transmission $L(n)$, and its $d(n)$ neighboring secondary user. In the following, we will use "user" to refer to any secondary user. A neighbor of a user $n$ is the user who shares similar spectrum availability, and will conflict with $n$ if both use the same channel. Note that there is a strong correlation between spectrum availability and user location, and users in close proximity are likely to share similar spectrum availability. We assume each user transmits using a predefined combination of operating parameters (power, modulation, etc.) and there is no power control. This leads to pseudo-static interference environment. We assume that each secondary user can track the spectrum usage of nearby primary users and obtain $L(n)$. Strategies to track interference condition and the number of conflicting neighbors $d(n)$ have been studied in [10].

We also assume that each channel has similar throughput capacity, e.g. 1. This is because that channel quality fluctuates due to fading, shadowing and environmental factors, making it impractical to collect channel quality of neighbors in real time. Hence, a reasonable approach is to assume all channels are identical in this respect. We are currently investigating the effects of non-uniform channel capacity on our algorithms.

\section{B. Rules for Conflict Free Channel Assignment}

We begin by describing rules that lead to conflict free spectrum use. Conflict free channel usage is one of the scheduling methods that allow for explicit and guaranteed throughput provisioning and control over packet delay. Such 
direct influence provides quality of service guarantees in an ad hoc network, especially for real-time applications.

We start with a simple rule: to prevent interference, users always select idle channels, i.e. channels unclaimed by neighbors. A channel is idle if the spectrum report shows no activity during the previous time period of length $X$, where $X$ is a design parameter. To provide fairness, we limit the number of channels each user can access.

Rule A (Uniform Idle Preference): Each user adjusts its spectrum usage to $\Omega=\min _{n}\left\lfloor\frac{L(n)}{d(n)+1}\right\rfloor$ idle channels.

Theorem 1: Rule A guarantees a conflict free spectrum allocation. (Proof in appendix).

The value of $\Omega$ can be set by a service provider to match network-wide conditions. However, a small number of users experiencing intensive interference from primary users (small $L(n)$ ) or other secondary users in a crowded area (large $d(n)$ ) can limit the the value of $\Omega$, leading to less than ideal spectrum utilization. Therefore, adapting $\Omega$ to each user's interference condition is preferred. Users in a crowded location should use a smaller number of channels compared to those in a sparse network. The work in [6] showed that collaborative spectrum allocation guarantees a minimum number of the channels each user $n$ can get. Referred to as the poverty line, $P L(n)=$ $\left\lfloor\frac{L(n)}{d(n)+1}\right\rfloor$, the bound is proportional to each user's interference condition. Related to the poverty line concept, we propose the following rule.

Rule B (Poverty Exact Idle Preference): A user $n$ selects exactly $P L(n)=\left\lfloor\frac{L(n)}{d(n)+1}\right\rfloor$ channels from idle channels. If number of idle channels $<P L(n)$, it "grabs" channels from "richer" users without impacting "poor" users. A user conflicting with a "poor" user will give up the channel and switch to other channels following the same procedure.

To $n$, a neighbor is "richer" if it uses more channels than $n$; otherwise it is "poor". Rule B requires that each user has knowledge of the number of neighbors $d(n)$, and the channel selection of each neighbor in order to identify "richer" users. To "grab" non-idle channels, a user $n$ marks the channels occupied by "poor" neighbors as busy, and the rest as idle. User $n$ then selects a set of channels from the "idle" channels until its channel occupancy reaches $P L(n)$.

A limitation of Rule B is that each user only attempts to use $P L(n)$ channels. Since $P L(n)$ represents a lower bound on spectrum usage derived using a collaboration based approach [6], Rule B could under-utilize available spectrum. Therefore, we devise an improved rule that guarantees the poverty line for each user while letting some users to go beyond their poverty lines.

Rule C (Poverty Guided Idle Preference): A user $n$ selects channels from idle channels. Only if there are not enough idle channels to reach $P L(n)$ does user n "grab" channels from "richer" neighbors. The number of channels it can grab from any "richer" user $r$, is $\min \{C(r)-P L(n), P L(n)-C(n)\}$ where $C(n)$ and $C(r)$ are the current spectrum usage of user $n$ and $r$.

Rule C allows users who have attained their poverty line to grab additional idle channels. It still allows users below their poverty line to grab channels from "richer" neighbors, but requires that each grabbing does not reduce a "richer" user's spectrum below the grabber's poverty line, avoiding cycles of users grabbing channels from each other in turn.

Assuming no two neighbors modify their spectrum/channel usage simultaneously, the system will reach equilibrium after a finite number of iterations. Equilibrium is the state where users have no incentive to adjust spectrum usage. The next theorem shows how Rule $\mathrm{C}$ provides the guarantees of Rule $\mathrm{B}$ while allowing higher utilization.

Theorem 2: Using Rule $B$ or $C$, the system reaches an equilibrium after at most $O\left(N^{2}\right)$ user spectrum modifications. In equilibrium, there is no conflict in spectrum usage, and each user's spectrum usage is no less than its Poverty Line $P L(n)$ (equal to $P L(n)$ for Rule $B)$. (Proof in appendix).

Additional mechanisms are required to implement Rule B and C. First, users (especially those below their poverty line) need to know the set of channels each neighbor currently occupies. This is done by each node broadcasting their channel usage either embedded in beacon broadcasts [24], or in routing hello messages [19]. These broadcasts are simple to implement, and their corresponding overhead is much smaller compared to that of collaboration based approaches [18], [6].

Second, Rule B and C assume no simultaneous spectrum adjustments by neighboring users. Since users can independently update their spectrum usage, a conflict occurs if two neighbors simultaneously switch to the same idle channel. Therefore, after a user decides to switch to a new channel, it computes a short random wait time before starting transmissions. If it detects activity on the channel during the wait time, it marks the channel as busy, and the channel switch is cancelled.

We note that the performance of conflict free channel assignments such as Rules B and C depends on the granularity of spectrum partition, i.e. the number of channels $M$. When $M$ is small compared to the number of neighbors $d(n)$, some users have a poverty line of zero, and hence no performance guarantee. In this case, the system can increase granularity by partitioning time and frequency together, e.g. a channel is defined as a frequency band at a particular time slot. One limitation is that this time slot approach requires tight time synchronization among users. Another option is to impose restrictions on device's duty cycle, e.g. each device can only stay alive for $p \%$ of time. This is roughly equivalent to increasing $M$ by $100 / p$. However, when multiple devices wake up concurrently, they need to contend for channels and avoid conflict. This requires additional rules.

\section{Rules for Contention-based Channel Assignment}

Broadcasting spectrum usage to neighbors might be undesirable for a number of reasons, including privacy concerns and 
protection against jamming from malicious users. For these reasons, we now describe several rules that do not require knowledge of neighbors' spectrum usage. When a user below its poverty line can not find any idle channel, we can either let users share channels through time slot scheduling [1], [20], [21], [22], or time-based random access [10], [13], [16]. Since time slot based approaches require time synchronization, we choose a random access based approach.

On each channel, users follow a set of random access rules such as CSMA to compete fairly for channel access and avoid conflict. Each user performs contention detection; listens to the channel before initiating any transmission. It initiates the transmission only when the channel is idle for some given time $T$. Otherwise, it backs off and delays the action for a short period. The value of $T$ and the backoff period are much smaller compared to the required idle time (defined as $X$ for Rule $\mathrm{A}, \mathrm{B}$ and $\mathrm{C}$ ). The penalty of such random access if the overhead of contention detection even if there is only user on the channel. It should be noted that since channels have different contention conditions, users should invoke independent contention detection and backoff process on each channel.

Random contention allows multiple users to share one channel but does not specify the number of channels users should use. Users could be selfish and occupy all the channels, reducing the system to a single channel with full interference. Therefore, we need to regulate the maximum number of channels each user can use.

Rule D (Selfish Spectrum Contention): Each user $n$ can use up to the $\Psi$ channels providing the highest throughput. Communication on each channel is through CSMA based time contention.

We note that the poverty line concept can provide a reference for choosing different value of $\Psi$ for different users. Since the poverty line represents throughput attainable from conflict free spectrum usage, $\Psi_{n}$ should be larger than $P L(n)$ to account for channel contention.

Rule E (Poverty Guided Selfish Spectrum Contention): This rule is the same as Rule D except the number of channels each user $n$ can use is limited by $\Psi_{n}=\max (\alpha \cdot P L(n), 1), \alpha \geq 1$.

Both rules encourage users to act selfishly. Users monitor channel conditions and switch to channels that provide the best throughput, even if such a switch might reduce performance for other neighbors. One question is how to choose the best channels with maximum capacity and minimum contention. For the purpose of illustration, we use the number of competing users as an indicator of channel quality. Hence, following Rule D or E, users always switch to channels with the least number of competing users. This also makes both rules efficient.

Theorem 3: Using Rule $D$ or $E$, the system will reach an equilibrium after at most $\Lambda \times M$ user spectrum modifications. $\Lambda$ is bounded by $O\left(N^{2}\right)$. (Proof in appendix).
The choice of $\Psi$ and $\alpha$ depends on specific random access mechanisms. To analyze their impact, we use a simple model to characterize channel sharing. When there are $m$ users sharing a channel, each user gets $\frac{1}{\lambda \cdot m}$ of channel throughput, where $\lambda$ is the contention penalty. When $\lambda=1.8$, this model matches the experimental test and analytical results for CSMA-based IEEE 802.11b systems at 11Mbps in [9].

We show that Rule D guarantees a lower bound on user throughput when $|L(n)|=M$ (See Theorem 4). The bound is only tight when $\Psi=M$, however, and cannot be used to derive the optimal value of $\Psi$. Theoretical analysis of Rules $\mathrm{D}$ and $\mathrm{E}$ is ongoing work.

Theorem 4: Using Rule $D$ and $(\lambda, m)$ model, a user $n$ 's throughput is bounded by $\operatorname{LB}(n, \Psi)=\frac{1}{\lambda\left\{\left\lfloor\frac{d(n)}{M}\right\rfloor+1\right\}},(\Psi=1)$; $\frac{m}{\lambda\left\{\left\lfloor\frac{\Psi \times d(n)}{M}\right\rfloor+2\right\}}, \quad(1<\Psi<M) ; \frac{M}{\lambda\{d(n)+1\}},(\Psi=M)$. (Proof in appendix)

\section{EXPERIMENTAL RESULTS}

We conduct experimental simulations to quantify the performance of the rule based spectrum access. We compare the performance of the rule based approaches to that of collaboration based approaches.

\section{A. Simulation Setup}

We assume a mobile ad hoc network by randomly placing users on $100 \times 100$ area, and use a binary interference metric - two users conflict if they are within distance of 20. This corresponds to the protocol interference model [11], [18]. That is, two distinct secondary users located within distance of $D_{s}$ are neighbors and will conflict if both use the same channel. Similarly, a secondary user and a primary user within distance of $D_{p}$ will conflict if both use the same channel. The values of $D_{s}$ and $D_{p}$ depend on signal strength of transmissions and decoding performance, thus are out of the scope of this paper. This model provides an approximation to the effects of interference in real wireless systems. It captures the way interference is manifested in wireless environments without delving into complex detection and decoding algorithms and protocols. We are currently investigating the impact of nonbinary interference metric on the proposed approach. In this paper, we simply set both $D_{s}$ and $D_{p}$ to 20 .

We investigate the performance with and without the presence of primary users. The default setting is without the presence of primary users. For networks without primary users, the spectrum is fully available to all secondary users. This also represents a scenario where nearby users see similar spectrum availability due to the wide coverage of a nearby primary user. For networks with primary users, we examine the average performance by randomly arranging the location of primary users. Both experiments show similar conclusions.

Each secondary user transmits data packets to some of its idle neighbors with constant power. Conflict due to uncoordinated transmission/reception is not considered. For simplicity, we assume that channels all have bandwidth of unit one. Figure 3 illustrates a sample topology of the simulated system 


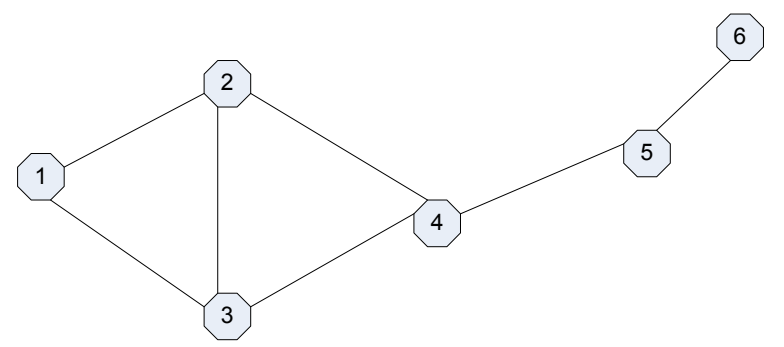

Fig. 3. A sample topology of the simulated system assuming 6 users.
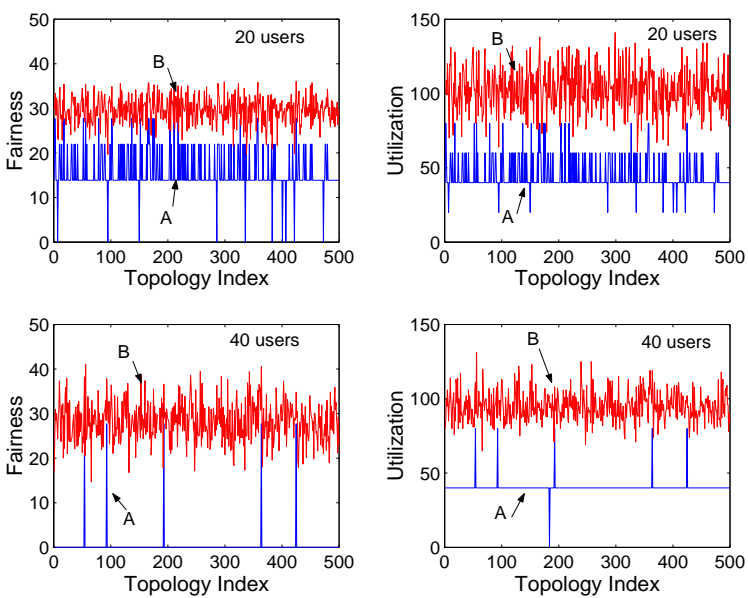

Fig. 4. Utilization comparison of Rule A and B assuming 20 channels and 20-40 users.

in terms of a conflict graph. Each vertex represents an active transmitter, and an edge exists between two vertices if they conflict. Mobility is simulated as in each time instance, $20 \%$ users move to another randomly selected location. Finally, we assume that the system can execute multiple adjustments and reach equilibrium inside the duration of a single time instance.

The spectrum usage is measured through two utility functions, utilization and fairness [25]. Assuming $\beta_{n}$ is user $n$ 's throughput over its selected channels, the utilization metric represents the total system throughput $\sum_{n} \beta_{n}$, and the fairness metric represents the level of proportional fairness $\sum_{n} \log \left(\beta_{n}\right)$. We run the experiments over 300 topologies, each topology with 200 time instances. For Rule C, D and E, the system has multiple equilibriums with different spectrum usage. We record the average performance over many equilibriums. Note that for the sake of comparison, we assume $\lambda=1$ for Rule D and E and set duty cycle to 1 .

\section{B. Comparison between Rules}

We first examine the effectiveness of poverty line based restrictions by comparing Rules $\mathrm{A}$ to $\mathrm{B}$ and $\mathrm{D}$ to $\mathrm{E}$. Each point on the $\mathrm{X}$-axis represents a single topology. Figure 4 shows that Rule B improves both the utilization and fairness by a factor of 2 over Rule A. Results in Figure 5 and 6 show that Rule E outperforms Rule D. In particular, Rule $\mathrm{D}$ is sensitive to the value of $\gamma$; small $\gamma$ leads to spectrum under-utilization while large $\gamma$ (aggressive spectrum usage)

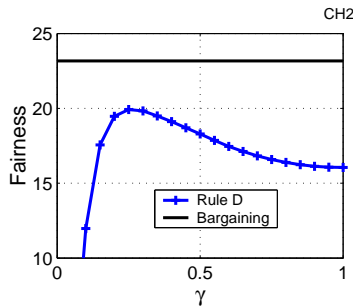

CH20UE40
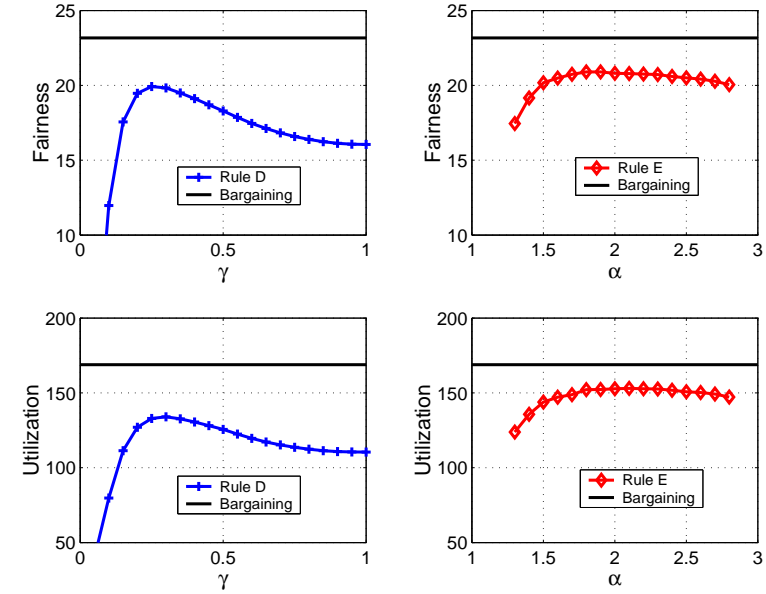

Fig. 5. Comparison of Rule $\mathrm{D}$ and $\mathrm{E}$ with bargaining scheme. $\gamma=\Psi / M$, average results of 40 users sharing 20 channels.

results in excessive interference. For the given configurations, $\gamma \in[0.3,0.5]$ provides the best performance. On the other hand, Rule $\mathrm{E}$ is relatively insensitive to the value of $\alpha$ and $\alpha>1.5$ provides better performance compared to Rule D.

In Figure 7, we compare the performance of rules B, C, $\mathrm{E}$ (with $\alpha=1.8$ ) assuming 40 users sharing 20 channels. The performance gap between Rule B and C shows that the poverty line is a still a loose bound on spectrum usage. By opportunistically going beyond the poverty line, users achieve better spectrum utilization. The performance difference between Rule $\mathrm{C}$ and $\mathrm{E}$ shows that Rule $\mathrm{E}$ provides better fairness, as $\alpha P L(n)$ provides a proportional increase in spectrum usage.

\section{Comparison to Collaboration based approaches}

Figure 7 also compares the rule-based approaches to collaboration-based approaches (CA), i.e. graph coloring and bargaining. We consider two graph coloring algorithms that maximize utilization and fairness respectively [18], [25], and a bargaining approach that maximizes fairness [6]. There is a noticeable performance gap between rule and collaboration based approaches. This confirms the effectiveness of using collaboration when power and complexity are not considered. Compared to the bargaining approach, Rule $\mathrm{C}$ leads to a graceful $8 \%$ degradation in utilization and $25 \%$ (on log-scale) in fairness.

Figure 8 summarizes the fairness and utilization of allocations derived from each multiple rule-based algorithms and the collaboration approach (CA). Note that fairness can degrade with the number of users for Rule B and $\mathrm{C}$, because of the effect of rounding down in the poverty line calculation.

\section{Algorithms Complexity and Communication Overhead}

For each of our five rules, Table 1 summarizes the information required at each user to execute the rule. They provide an intuitive measure of each rule's required communication complexity. 


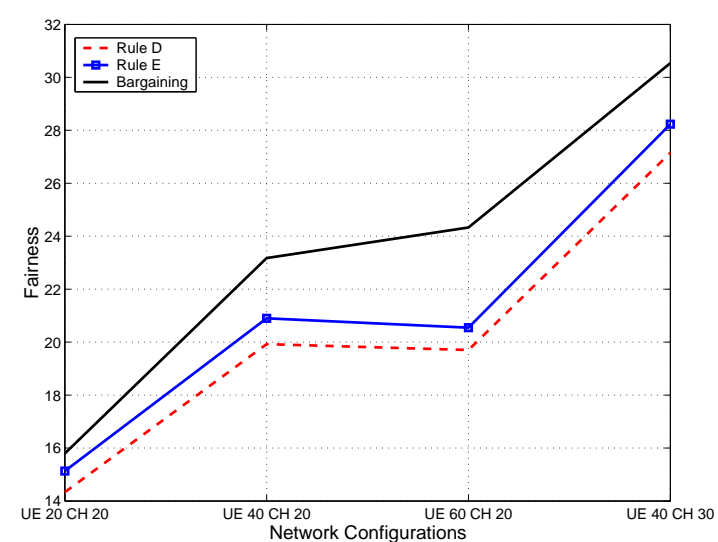

(a)

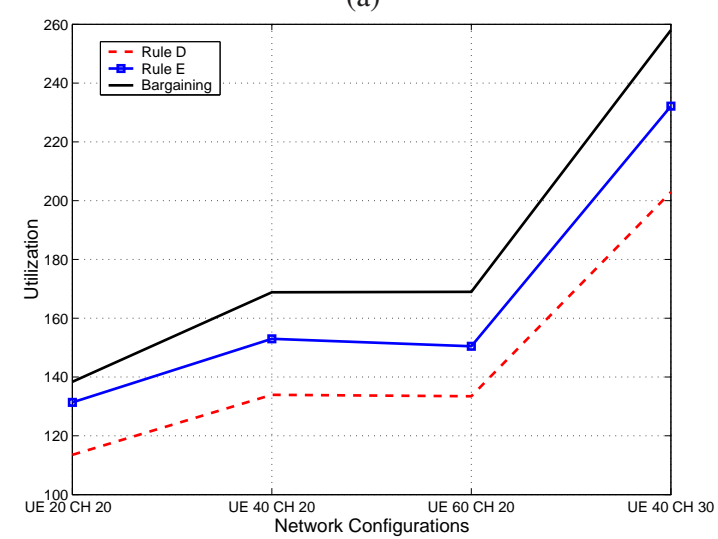

(b)

Fig. 6. Comparison of Rule D and E with bargaining scheme. $\gamma=\Psi / M$. (a) Fairness under different network configurations. Rule E with $\alpha=1.8$ and Rule D with optimal $\gamma$ (b) Utilization under different network configurations. Rule E with $\alpha=1.8$ and Rule D with optimal $\gamma$.

\begin{tabular}{|c|c|c|c|c|}
\hline & $\begin{array}{c}\text { channel } \\
\text { idle/busy }\end{array}$ & $\begin{array}{c}\text { No. neighbors } \\
d_{n}\end{array}$ & $\begin{array}{c}\text { each neighbor's } \\
\text { channel usage }\end{array}$ & $\begin{array}{c}\text { channel } \\
\text { utilization }\end{array}$ \\
\hline Rule A & $\checkmark$ & & & \\
\hline Rule B & $\checkmark$ & $\checkmark$ & $\checkmark$ & \\
\hline Rule C & $\checkmark$ & $\checkmark$ & $\checkmark$ & \\
\hline Rule D & $\checkmark$ & & & $\checkmark$ \\
\hline Rule E & $\checkmark$ & $\checkmark$ & & $\checkmark$ \\
\hline
\end{tabular}

TABLE I

REQUIRED INFORMATION TO EXECUTE THE RULES

In Figure 9, we measure the complexity of different algorithms in terms of the total number of spectrum adjustments before the system reaches an equilibrium. We use the default network configuration with 20 channels and 40 secondary users. Compared to the bargaining approach, Rule $\mathrm{C}$ provides more than $60 \%$ reduction in complexity, and thus much faster adaptation to topology changes. Rule E offers similar number of adjustments as the bargaining approach.

The communication overhead can be derived based on the number of spectrum adjustments. For the collaboration based approaches (the graph coloring and bargaining), a 4-way handshaking is required for each spectrum adjustment, so that the message overhead is 4 times the number of adjustments. For

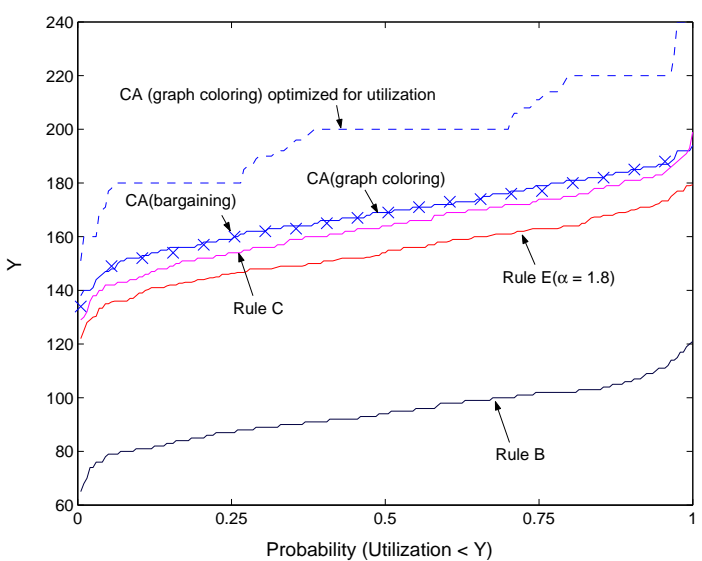

(a) Utilization

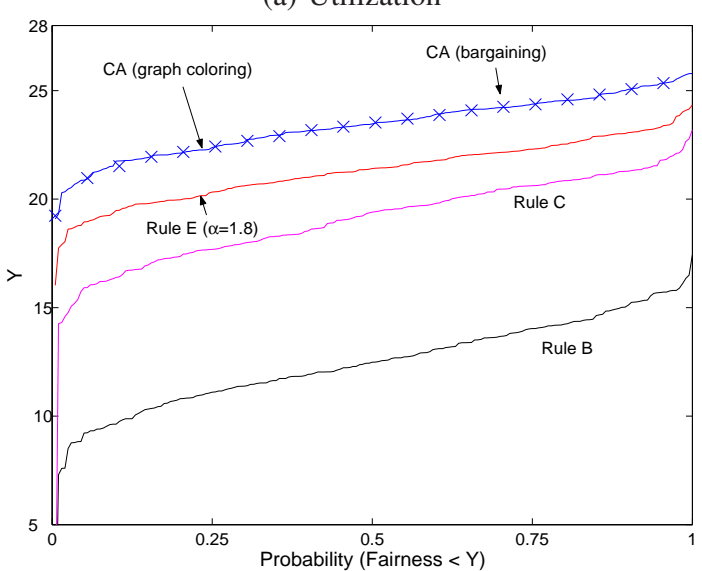

(b) Fairness

Fig. 7. Utilization and Fairness comparison of different rules for 20 channels and 40 users.

Rule C, each user broadcasts its spectrum usage to neighbors after the adjustment, resulting in one message per adjustment. In terms of coordination overhead, Rule C leads to $240 \%$ reduction compared to the bargaining approach. Considering its minor degradation in system utility and significant reduction in complexity, Rule $\mathrm{C}$ is a good low-complexity alternative to collaboration based approaches. For Rule E, if each user can measure channel utilization effectively, no message exchange is required. Given its low communication requirements, Rule $\mathrm{E}$ is a low-communication alternative to both Rule $\mathrm{C}$ and the collaboration based approaches.

Figure 10 shows that the number of spectrum adjustments required to reach equilibrium scales linearly with the number of users. This further demonstrates the efficiency of the rulebased spectrum management approach.

\section{E. Impact of Primary Users}

We now examine spectrum usage in the presence of primary users. We randomly deploy a set of primary users in the network, each occupying a channel. Each secondary user obtains its spectrum availability and follows the rule to access spectrum bands. In Figure 11, we measure the fairness and utilization of allocations derived from Rules $\mathrm{C}$ and $\mathrm{E}$ and the 

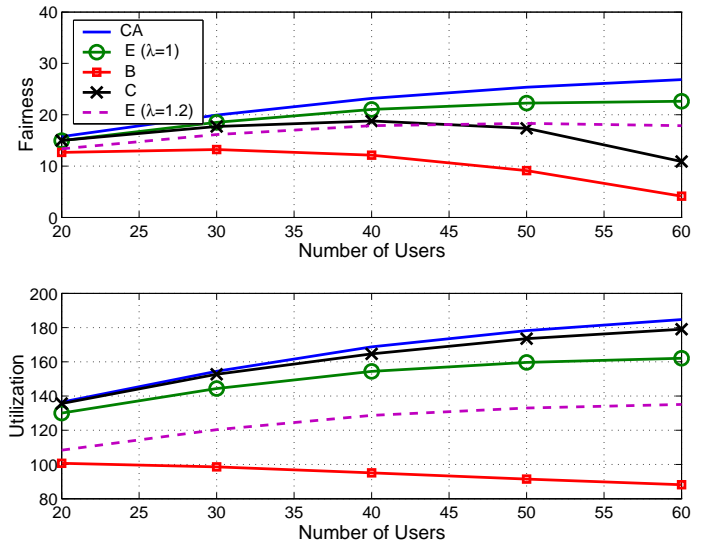

Fig. 8. Utilization and Fairness comparison of different rules assuming 20 channels.

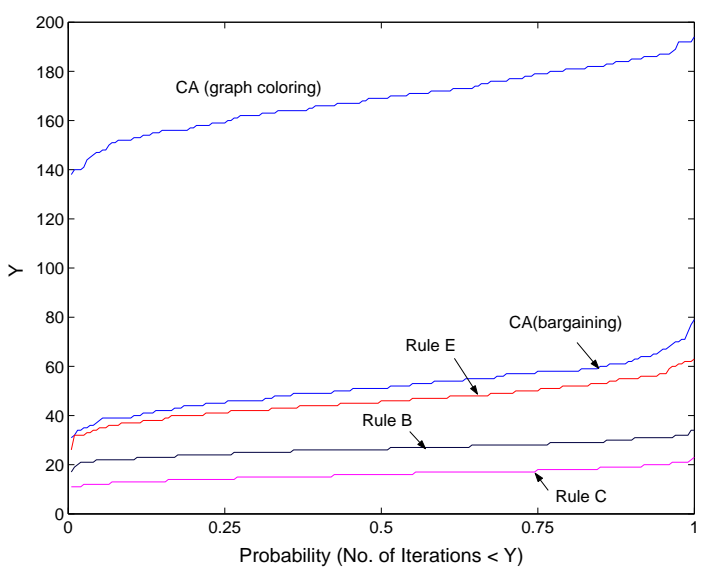

Fig. 9. Complexity (the number of spectrum adjustments) comparison of different rules for 20 channels and 40 users.

bargaining based collaborative approach (CA), assuming 40 secondary users, 20 spectrum channels and different number of primary users. The utilities are averaged over 100 randomly deployment of primary users. Similar conclusions are drawn. The performance of both rule and collaboration based approaches degrade gracefully as the number of primary users increases.

\section{Discussions AND Future WORK}

Practical usage of the proposed rules depends on the characteristics of the application. Conflict-free assignment based Rules A, B and C let users reserve channels, and are preferable when the spectrum is finely partitioned, and when users have constant traffic, e.g. video streaming. Contention-based rules (Rules D and E) require fine granularity in time to efficiently utilize spectrum, providing improved utilization for coarsely partitioned spectrum and bursty data traffic. The penalty is contention overhead and collisions.

We did not discuss user behavior under faulty conditions. Under conflict-free rules (A, B, C), a user can broadcast alarm signals when it can not get its guaranteed spectrum under the rules. The alarm triggers fault-handlers in other users, causing
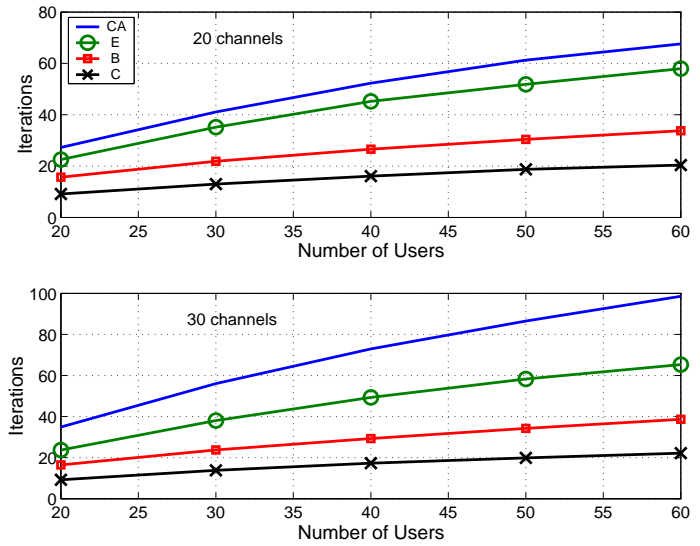

Fig. 10. Complexity (the number of spectrum adjustments) comparison of different rules.

them to check their own configurations. The detailed design and the mechanisms for protection against malicious users are out of the scope of this paper, but are important open research areas.

In this paper, we focus on the task of dynamic spectrum selection which is in general a responsibility of MAC layer. We are aware that modifications are required at higher layers to respond to dynamically changing spectrum availability among secondary users. In particular, we did not discuss behavior of multi-hop transmissions where destination user is out of the transmission range of source user, and packets are routed to the destination by users in between. Route selection impacts the traffic load on each link and the amount of spectrum/bandwidth required. Joint selection of route and spectrum could make better usage of spectrum and improve end-to-end performance for multi-hop transmissions. We are currently investigating a spectrum aware routing protocol that adapts route selection to spectrum fluctuations.

\section{CONCLUSION}

For open spectrum systems on resource-constrained networks, we propose a device-centric spectrum management scheme where users act independently based on local observations and spectrum rules, resulting in significantly lower communication between users. We propose five rules that tradeoff performance with implementation and communication complexity. We show that rules guided by a lower-bound calculation (poverty line) provide superior performance. Experimental results show that rule-based approaches perform slightly worse than the previously proposed collaborative approaches, but have much lower complexity and communication overhead.

\section{REFERENCES}

[1] Bao, L., And Garcia-Luna-Aceves, J. J. Hybrid channel access scheduling in ad hoc networks. In Prof. ICNP (2002).

[2] Berger, R. J. Open spectrum: a path to ubiquitous connectivity. ACM Queue 1, 3 (May 2003).

[3] Berlemann, L., Walke, B., And Mangold, S. Behavior based strategies in radio resource sharing games. In Proc. of IEEE PIMRC (Nov. 2004). 


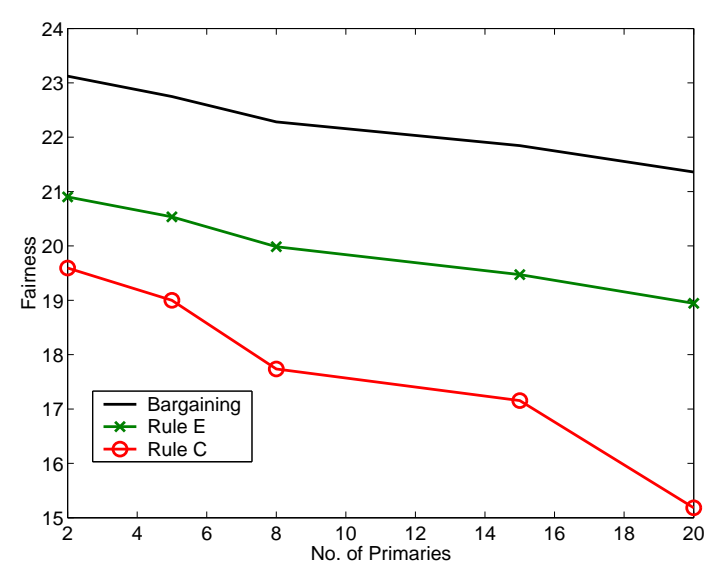

(a) Fairness

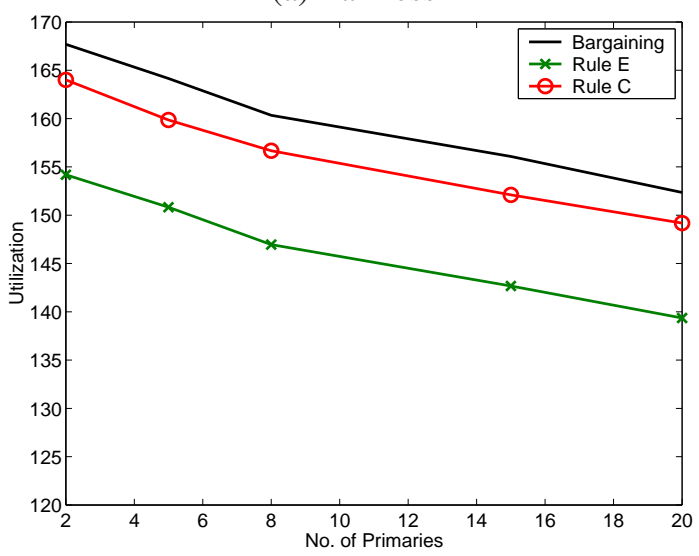

(b) Utilization

Fig. 11. Utilization and Fairness comparison of different rules assuming 20 channels, 40 secondary users and 20 primary users.

[4] Brodersen, R. W., Wolisz, A., Cabric, D., Mishra, S. M., ANd WILLKOMM, D. Corvus: a cognitive radio approach for usage of virtual unlicensed spectrum. Whitepaper, July 2004.

[5] CAO, L., AND Zheng, H. A game theory approach towards fair spectrum sharing. work in process.

[6] CAO, L., And Zheng, H. Distributed spectrum allocation via local bargaining. In IEEE SECON (Santa Clara, CA, Sept. 2005).

[7] Ephremides, A., AND Truong, T. Scheduling broadcasts in multihop radio networks. In IEEE Trans. on Communications (April 1990), vol. 38 , pp. 456-460.

[8] HAN, Z., Ji, Z., AND LiU, K. R. Low-complexity OFDMA channel allocation with Nash bargaining solution fairness. In IEEE GLOBECOM '04 (2004).

[9] Heusse, M., Rousseau, F., Berger-Sabbatel, G., And Duda, A. Performance anomaly of 802.11 b. In Proc. of INFOCOM (2003).

[10] Huang, X., AND B.Bensaou. On max-min fairness and scheduling in wireless ad-hoc networks: analytical framework and implementation. In Proc. of Mobihoc (2001), ACM.

[11] Jain, K., Padhye, J., V.N.Padmanabha, AND QiU, L. Impact of interference on multi-hop wireless network performance. In Proc. ACM Mobicom (2003).

[12] Katzela, I., AND Naghshineh, M. Channel assignment schems for celluar mobile telecommunication systems. IEEE Personal Communications 3, 3 (June 1996), 10-31.

[13] Luo, H., Lu, S., And Bharghavan, V. A new model for packet scheduling in multihop wireless networks. In Proc. ACM Mobicom (August 2000).

[14] Mangold, S., Zhong, Z., Challapali, K., and Chou, C. T. Spectrum agile radio: Radio resource measurements for opportunistic spectrum usage. In Proc. of IEEE Globecom (Nov. 2004)
[15] McHenry, M. Spectrum white space measurements. New America Foundation Broadband Forum (June 2003).

[16] Nandagopal, T., T.Kim, X.Gao, and Bharghavan, V. Achieving mac layer fairness in wireless packet networks. In Proc. ACM Mobicom (August 2000).

[17] PeHA, J. M. Approaches to spectrum sharing. In IEEE Communications Magazine (Feb. 2005).

[18] Peng, C., Zheng, H., And ZhaO, B. Y. Utilization and fairness in spectrum assignemnt for opportunistic spectrum access. Mobile Networks and Applications (MONET) (2006). to appear.

[19] Perkins, C., AND Royer, E. Ad hoc on-demand distance vector routing. In Proc. of WMCSA (February 1999), ACM.

[20] Ramanathan, S. A unified framework and algorithm for channel assignment in wireless networks. Wireless Networks 5, 2 (March 1999), 81-94.

[21] Ramanathan, S., AND Lloyd, E. Scheduling algorithms for multihop radio networks. In IEEE/ACM Trans. on Networking (April 1993), vol. 1, pp. 166-177.

[22] Salonidis, T., and Tassiulas, L. Distributed on-line schedule adapation for balanced slot allocation in wireless ad hoc networks. In Proc. of IWQoS (2004).

[23] XG working group RFC, the $\mathrm{XG}$ vision and the $\mathrm{XG}$ architecture. http://www.darpa.mil/ato/programs/XG.

[24] ZHAO, J., Zheng, H., AND YANG, G. Distributed coordination in dynamic spectrum allocation networks. Accepted by Dyspan, Baltimore, Nov. 2005.

[25] Zheng, H., AND Peng, C. Collaboration and fairness in opportunistic spectrum access. In Proc. 40th annual IEEE International Conference on Communications (June 2005).

\section{APPENDIX A: Proof of THEOREMS}

\section{A. Proof of Theorem 1}

A user $i$ observes its neighbors' spectrum usage when selecting channels. The total number of channels occupied by its neighbors is at most $O(i)=\Omega \cdot d(i)$. Obviously, $\Omega \leq L(i)-O(i)$. Hence, user $i$ can always find at least $\Omega$ idle channels that will not lead to any conflict.

\section{B. Proof of Theorem 2}

First, we show that using Rule B or $\mathrm{C}$, the following properties hold for each user.

Property 1: A node $n$ can find at least $P L(n)$ channels, which do not lead to conflict with neighbors whose poverty line is equal or less than that of $n$.

Proof: [Proof for Rule B] There are at most $d(n)$ neighbors of $n$ with less or equal Poverty Line than $n$, denoted $n_{1}, n_{2}, \cdots, n_{i}, i \leq d(n)$. Then $\left|\bigcup_{s=1}^{i} C\left(n_{s}\right)\right| \leq$ $\sum_{s=1}^{i}\left|C\left(n_{s}\right)\right| \leq d(n) \times P L(n) \leq \frac{d(n)}{d(n)+1} L(n)$, where $C(n)$ represents the spectrum usage of user $n$. So at least $(1-$ $\left.\frac{d(n)}{d(n)+1} L(n)\right) \geq P L(n)$ channels are available for $n$ to use.

Proof: [Proof for Rule C] A user $n$ can reserve $P L(n)$ channel(s) for each neighbor, regardless of their Poverty Line. Note that Rule $\mathrm{C}$ does not require each user to have knowledge of its neighbors' poverty line. It is straightforward to show that the number of remaining channels for $n$ is larger than $P L(n)$. Following Rule $\mathrm{C}$, when a user $n$ conflicts with a neighbor with equal or less poverty line than $P L(n), n$ will give up the channel(s) that are not reserved for it by its neighbor and switch to other channel(s). The property follows. 
We define a user as "qualified" if its channel usage does not conflict with any of its neighbors with poverty line equal or less than that of user $n$. Otherwise, the user is "disqualified". Based on Rule B and C, only "disqualified" users modify their channel usage. Based on Property 1, after modifications, a "disqualified" user becomes "qualified". Furthermore, the following property holds.

Property 2: When a user $n$ modifies its channel usage and transfers any of its neighbors, i.e. user $n_{1}$ from "qualified" to "disqualified", then $P L(n)<P L\left(n_{1}\right)$.

Proof: Note that we assume only $n$ is modifying its channel usage. If $P L(n) \geq P L\left(n_{1}\right)$, then after the modification $n$ won't conflict with $n_{1}$, so $n_{1}$ won't change from "qualified" to "disqualified".

Proof: [Proof for Theorem 2] At an equilibrium, each user has no incentive to modify its spectrum usage. By Property 1 , it is straightforward that at an equilibrium, for all $n$, $|C(n)| \geq P L(n)$, and the channel selection of $n$ does not conflict with its neighbors with equal or lower Poverty Line compared to $P L(n)$. Hence, the whole system is conflict-free and each user's spectrum usage reaches its Poverty Line.

If spectrum modification is conducted in a way where a user with lower poverty line than its neighbors modifies its channel usage first, then each "qualified" user will never become a "disqualified" user after its own spectrum modification. Hence, the number of iterations is at most $N$. When spectrum modification is disordered, we can use induction to prove that the maximum number of iterations is bounded by $O\left(N^{2}\right)$. The detailed proof will be included in a future paper [5].

\section{Proof of Theorem 3}

We start the proof by defining a conflict graph $G=\{U, E\}$, where $U$ represents the list of vertices (the secondary users), $(|U|=N)$, and $E$ represents the edges between vertices. An edge exists between two vertices if the corresponding users conflict with each other if using the same channel. $\Lambda=|E|$ represents the number of edges in the conflict graph. It is obvious that the number of edges is maximized when the network is all connected, where $\Lambda=N(N-1) / 2$.

Let $A$ characterize the network's spectrum usage, i.e. the channel(s) used by each secondary user(vertex). We now define the conflict factor of $A$ as

$$
C F(A)=\sum_{m=0}^{M-1} C F_{m}(A),
$$

where $C F_{m}(A)$ is the number of the pair of conflicting users who are using channel $m$.

Following Rules D and E, a user $n$ switches from channel $m$ to $m^{\prime}$ only if it can gain more throughput from $m^{\prime}$ than from $m$. Based on the interference model, the number of users conflicting with $n$ on channel $m^{\prime}$ is smaller than that on channel $m$. Therefore, we can derive the following.

$$
\begin{array}{r}
C F_{m^{\prime}}\left(A^{\prime}\right)-C F_{m^{\prime}}(A)<C F_{m}(A)-C F_{m}\left(A^{\prime}\right) \Rightarrow \\
C F_{m^{\prime}}\left(A^{\prime}\right)+C F_{m}\left(A^{\prime}\right)<C F_{m}(A)+C F_{m^{\prime}}(A) .
\end{array}
$$

Since the switch will only impact on the values of $C F_{m}($. and $C F_{m^{\prime}}($.$) , it is obvious that C F\left(A^{\prime}\right)<C F(A)$. Hence, $C F$ strictly decreases each time a user modifies its channel usage. $C F(A) \leq|E| \times M$ and $C F \in \mathcal{Z}$, the switch will end after at most $|E| \times M$ iterations, and the system will reach an equilibrium.

\section{Proof of Theorem 4}

Proof for $\Psi=1$ : By Pigeonhole Principle, there must exist a channel where a user $n$ observes less or equal than $\left\lfloor\frac{d(n)}{M}\right\rfloor$ neighbors. At an equilibrium, each user selfishly selects the best channel for itself, and conflicts with at most $\left\lfloor\frac{d(n)}{M}\right\rfloor$ neighbors.

Proof for $1<\Psi<M$ : assuming that a user $n$ is assigned with $m$ channels. By an inductive argument using Pigeonhole Principle, there must exist $m$ channels, denoted $c_{1}, c_{2}, \cdots, c_{m}$, such that

$$
d_{c_{1}}(n)+d_{c_{2}}(n)+\cdots+d_{c_{m}}(n) \leq m \times\left(\left\lfloor\frac{m \times d(n)}{M}\right\rfloor+1\right) .
$$

Here we use $d_{c}(n)$ to denote the number of neighbors of $n$ who are occupying channel $c$. Now if user $n$ switches to these $m$ channels, its utility will be

$$
\begin{aligned}
& \frac{1}{\lambda}\left(\frac{1}{d_{c_{1}}(n)+1}+\frac{1}{d_{c_{2}}(n)+1}+\cdots+\frac{1}{d_{c_{m}}(n)+1}\right) \\
& \geq \frac{1}{\lambda} \frac{m}{\left(\left(d_{c_{1}}(n)+1\right)\left(d_{c_{2}}(n)+1\right) \cdots\left(d_{c_{m}}(n)+1\right)\right)^{\frac{1}{m}}} \\
& \geq \frac{m}{\lambda} \frac{m}{\left(\frac{\left(d_{c_{1}}(n)+1\right)+\left(d_{c_{2}}(n)+1\right)+\cdots+\left(d_{c_{m}}(n)+1\right)}{m}\right)^{m \frac{1}{m}}} \\
& =\frac{1}{\lambda} \frac{m^{2}}{d_{c_{1}}(n)+1+d_{c_{2}}(n)+1+\cdots+d_{c_{m}}(n)+1} \\
& \geq \frac{1}{\lambda} \frac{m^{2}}{m\left(\left\lfloor\frac{m d(n)}{M}\right\rfloor+1\right)+m} \\
& =\frac{m}{\lambda\left\{\left\lfloor\frac{m d(n)}{M}\right\rfloor+2\right\}} .
\end{aligned}
$$

Proof for $\Psi=M$ : straightforward following the interference model. For each user, there are $d(n)+1$ users competing for all $M$ channels. 